\title{
Antimicrobial susceptibility among gram-negative isolates collected in the USA between 2005 and 2011 as part of the Tigecycline Evaluation and Surveillance Trial (T.E.S.T.)
}

Gerald A Denys ${ }^{1 *}$, Steven M Callister ${ }^{2}$ and Michael J Dowzicky ${ }^{3}$

\begin{abstract}
Background: The Tigecycline Evaluation and Surveillance Trial (T.E.S.T.) was designed to monitor in vitro antimicrobial susceptibility to tigecycline and comparator agents. We present susceptibility data on Gram-negative organisms collected between 2005 and 2011 from nine United States census regions.

Methods: T.E.S.T. was conducted using standardized CLSI methodologies or FDA-approved breakpoints.

Results: Tigecycline was highly active $\left(\mathrm{MIC}_{90} \leq 2 \mathrm{mg} / \mathrm{L}\right)$ against Enterobacteriaceae irrespective of species or region of collection ( $N=25011)$. The isolates were also highly susceptible to the carbapenems when all regional data are combined, except for ESBL-producing Klebsiella pneumoniae ( $\mathrm{MIC}_{90} 16 \mathrm{mg} / \mathrm{L}$ ) and Acinetobacter baumannii ( $\mathrm{MIC}_{90}$ $\geq 32 \mathrm{mg} / \mathrm{L}$ ). In addition, 883 (30\%) of 2900 A. baumannii isolates were classified as multidrug-resistant (MDR): these MDR organisms were most susceptible to tigecycline ( MI $_{90} 2 \mathrm{mg} / \mathrm{L}$ ) and minocycline ( $M I C_{90} 8 \mathrm{mg} / \mathrm{L}$ ) when all regional data are considered together. Susceptibility patterns also varied widely among the regions.
\end{abstract}

Conclusions: The findings highlight the importance of monitoring antimicrobial susceptibility patterns and implementing effective methods to curb increased resistance and also confirm that additional studies to determine the efficacy of tigecycline in vivo, especially for treating infections with MDR organisms, are warranted.

Keywords: Surveillance, Tigecycline, Resistance, USA, Census regions

\section{Background}

Infection with Acinetobacter spp. and some members of the Enterobacteriaceae present clinicians with considerable challenge, especially since resistance to carbapenems is becoming increasingly prevalent $[1,2]$. Such infections result in increased mortality and morbidity, and the increased hospitalization costs continue to put enormous strain on the healthcare system [3-5]. Against this background, surveillance studies designed to monitor antimicrobial resistance of Gram-negative bacteria collected from regions throughout the USA are essential.

Tigecycline is a novel glycylcycline antimicrobial that overcomes several common mechanisms used by bacteria to develop resistance [6]. Confirmation of the effectiveness

\footnotetext{
* Correspondence: gdenys@iuhealth.org

'Indiana University Health Pathology Laboratory, Indianapolis 46202, IN, USA Full list of author information is available at the end of the article
}

of tigecycline against a broad spectrum of microorganisms resulted in licensing by the US Food and Drug Administration (FDA) for the treatment of complicated skin and skin structure infections (cSSSIs), intra-abdominal infections (cIAIs), and community-acquired bacterial pneumonia (CAP) [7].

The Tigecycline Evaluation and Surveillance Trial (T.E. S.T.) is a multi-center study designed to monitor the in vitro activity of tigecycline and a wide range of other antimicrobial agents against clinically-important Grampositive and Gram-negative pathogens collected globally. This report focuses on data obtained from Gram-negative organisms collected in the USA. In a previous report, Halstead et al. [8] confirmed significant in vitro activity of tigecycline against $A$. calcoaceticus-baumannii complex, Enterobacter spp., Escherichia coli, and Klebsiella pneumoniae isolates and highlighted the ability of local 
susceptibility patterns to more effectively guide empiric antimicrobial therapy. This study reports the in vitro activity of tigecycline against $A$. baumannii and other Enterobacteriaceae isolates. In addition, the susceptibility patterns among nine distinct regions within the USA are presented and emerging trends in resistance are evaluated by comparing the results to previous findings $[8]$.

\section{Methods}

\section{Isolate collection}

Gram-negative isolates were collected between 2005 and 2011 from 173 centers that were divided into nine census regions: East North Central, 31; East South Central, 11; Middle Atlantic, 44; Mountain, 7; New England, 7; Pacific, 11; South Atlantic, 33; West North Central, 16; West South Central, 13. The states contained in each census region are shown in Table 1.

The centers submitted clinically-significant (determined by local criteria) Gram-negative isolates that were collected consecutively. The organisms included Acinetobacter spp., E. coli, Enterobacter spp., Serratia spp., and Klebsiella spp. A single isolate was collected from each patient and inclusion was independent of medical history, previous antimicrobial use, sex or age of the patient.

Organisms were identified using routine methodologies practiced regularly at each institution. Prior to eligibility for participation, the medical centers were evaluated by the central laboratory (IHMA: Laboratories International for Microbiology Studies, a division of International Health Management Associates, Inc. [IHMA, Schaumburg, IL, USA]) for adherence to national guidelines. In addition, IHMA confirmed the identification of organisms or antimicrobial susceptibility patterns using RapidOne and/or RapidNF identification systems (Remel, Lenexa, KS) when the forwarded results were uncharacteristic.

\section{Susceptibility testing}

Minimum inhibitory concentrations (MICs) were determined using broth microdilution methodology, Sensititre ${ }^{\circ}$ plates (TREK Diagnostic Systems, West Sussex, England) or MicroScan panels (Siemens, Sacramento, CA, USA). Susceptibility to amikacin, amoxicillin-clavulanate, ampicillin, carbapenems (imipenem/meropenem), cefepime, ceftazidime, ceftriaxone, levofloxacin, minocycline, and piperacillin-tazobactam were interpreted according to the guidelines published by the Clinical and Laboratory Standards Institute [9-11]. In addition, susceptibility to imipenem was evaluated on isolates collected through 2006; meropenem was substituted thereafter due to imipenem stability issues. MIC values against tigecycline were evaluated using FDA-approved breakpoints provided in the package insert [7]. Quality control strains were $E$. coli ATCC 25922 and P. aeruginosa ATCC 27853. Results are presented as $\mathrm{MIC}_{90}$.

\section{Extended-spectrum $\beta$-lactamase (ESBL) testing}

Extended spectrum $\beta$-lactamase (ESBL) production by $E$. coli or Klebsiella spp. was also confirmed using accepted methodology [10]. Briefly, discs that contained cefotaxime $(30 \mu \mathrm{g})$, cefotaxime/clavulanic acid $(30 / 10 \mu \mathrm{g})$, ceftazidime $(30 \mu \mathrm{g})$, or ceftazidime/clavulanic acid $(30 / 10 \mu \mathrm{g})$ (Oxoid, Inc., Ogdensburg, NY, USA) were placed onto MuellerHinton agar (Remel, Inc., Lenexa, KS) plates after they were overlaid with the isolate. Organisms where the combination of cefotaxime and ceftazidine discs yielded a zone of inhibition larger by $>5 \mathrm{~mm}$ than the zone of inhibition for cefotaxime or ceftazidime were considered ESBLproducers. K. pneumoniae ATCC 700603 (ESBL-positive)

Table 1 Numbers of isolates contributed by census region ${ }^{a}$ in T.E.S.T.

\begin{tabular}{lcccccccccc}
\hline Pathogen & Pacific & Mountain & $\begin{array}{c}\text { West North } \\
\text { Central }\end{array}$ & $\begin{array}{c}\text { East North } \\
\text { Central }\end{array}$ & $\begin{array}{c}\text { Middle } \\
\text { Atlantic }\end{array}$ & $\begin{array}{c}\text { New } \\
\text { England }\end{array}$ & $\begin{array}{c}\text { South } \\
\text { Atlantic }\end{array}$ & $\begin{array}{c}\text { East South } \\
\text { Central }\end{array}$ & $\begin{array}{c}\text { West South } \\
\text { Central }\end{array}$ & $\begin{array}{c}\text { Total } \\
\text { A. baumannii }\end{array}$ \\
\hline 103 & 72 & 332 & 694 & 721 & 77 & 562 & 162 & 177 & 2900 \\
E. coli & 240 & 235 & 813 & 1477 & 1892 & 226 & 1248 & 391 & 398 & 6920 \\
K. pneumoniae & 181 & 164 & 590 & 1174 & 1518 & 170 & 1096 & 331 & 311 & 5535 \\
K. oxytoca & 57 & 50 & 176 & 301 & 279 & 45 & 145 & 49 & 68 & 1170 \\
S. marcescens & 87 & 61 & 302 & 544 & 609 & 67 & 475 & 140 & 136 & 2421 \\
Enterobacter spp. & 227 & 192 & 770 & 1351 & 1500 & 198 & 1118 & 339 & 370 & 6065 \\
Total & 895 & 774 & 2983 & 5541 & 6519 & 783 & 4644 & 1412 & 1460 & 25011 \\
\hline
\end{tabular}

${ }^{a}$ Pacific = California, Hawaii, Oregon, and Washington; Mountain = Arizona, Colorado, New Mexico, Montana, and Utah; West North Central = lowa, Kansas, Minnesota, Missouri, Nebraska, North Dakota, and South Dakota; East North Central = Indiana, Illinois, Michigan, Ohio, and Wisconsin; Middle Atlantic = New Jersey, New York, and Pennsylvania; New England = Connecticut, Maine, Massachusetts, New Hampshire, and Vermont; South Atlantic = Delaware, District of Columbia, Florida, Georgia, Maryland, North Carolina, South Carolina, Virginia, and West Virginia; East South Central = Alabama, Kentucky, Mississippi, and Tennessee; West South Central = Arkansas, Louisiana, Oklahoma, and Texas. 
Table 2 Antimicrobial susceptibility for Acinetobacter baumannii and multidrug-resistant (MDR) A. baumannii

\begin{tabular}{|c|c|c|c|c|c|c|c|c|c|c|c|}
\hline & & Pacific & Mountain & $\begin{array}{l}\text { West North } \\
\text { Central }\end{array}$ & $\begin{array}{l}\text { East North } \\
\text { Central }\end{array}$ & $\begin{array}{l}\text { Middle } \\
\text { Atlantic }\end{array}$ & $\begin{array}{c}\text { New } \\
\text { England }\end{array}$ & $\begin{array}{c}\text { South } \\
\text { Atlantic }\end{array}$ & $\begin{array}{l}\text { East South } \\
\text { Central }\end{array}$ & $\begin{array}{l}\text { West South } \\
\text { Central }\end{array}$ & USA \\
\hline $\begin{array}{l}\text { Acinetobacter } \\
\text { baumannii }\end{array}$ & & $N=103$ & $N=72$ & $N=332$ & $N=694$ & $N=721$ & $N=77$ & $N=562$ & $N=162$ & $N=177$ & $N=2900$ \\
\hline \multirow[t]{3}{*}{ Amikacin } & $\mathrm{MIC}_{50}$ & 4 & 4 & 4 & 8 & 4 & 4 & 4 & 4 & 4 & 4 \\
\hline & $\mathrm{MIC}_{90}$ & $\geq 128$ & $\geq 128$ & $\geq 128$ & $\geq 128$ & 64 & 8 & 64 & $\geq 128$ & $\geq 128$ & $\geq 128$ \\
\hline & $\% \mathrm{~S}$ & 71.8 & 69.4 & 80.4 & 58.8 & 78.1 & 100 & 85.1 & 69.8 & 71.2 & 74.3 \\
\hline \multirow[t]{3}{*}{ Carbapenems } & $\mathrm{MIC}_{50}$ & 1 & 1 & 1 & 4 & 1 & 1 & 1 & 2 & 1 & 1 \\
\hline & $\mathrm{MIC}_{90}$ & 16 & $\geq 32$ & $\geq 32$ & $\geq 32$ & $\geq 32$ & $\geq 32$ & $\geq 32$ & $\geq 32$ & $\geq 32$ & $\geq 32$ \\
\hline & $\% \mathrm{~S}$ & 76.7 & 69.4 & 74.7 & 50.3 & 70.3 & 74.0 & 74.0 & 66.7 & 80.2 & 67.4 \\
\hline \multirow[t]{3}{*}{ Cefepime } & $\mathrm{MIC}_{50}$ & 16 & 8 & 8 & 16 & 16 & 16 & 8 & 32 & 8 & 16 \\
\hline & $\mathrm{MIC}_{90}$ & $\geq 64$ & $\geq 64$ & $\geq 64$ & $\geq 64$ & $\geq 64$ & $\geq 64$ & $\geq 64$ & $\geq 64$ & $\geq 64$ & $\geq 64$ \\
\hline & $\% \mathrm{~S}$ & 46.6 & 55.6 & 55.7 & 38.2 & 42.7 & 48.1 & 52.8 & 40.1 & 55.9 & 46.3 \\
\hline \multirow[t]{3}{*}{ Ceftazidime } & $\mathrm{MIC}_{50}$ & 16 & $\leq 8$ & $\leq 8$ & $\geq 64$ & 32 & 16 & 16 & $\geq 64$ & $\leq 8$ & 32 \\
\hline & $\mathrm{MIC}_{90}$ & $\geq 64$ & $\geq 64$ & $\geq 64$ & $\geq 64$ & $\geq 64$ & $\geq 64$ & $\geq 64$ & $\geq 64$ & $\geq 64$ & $\geq 64$ \\
\hline & $\% \mathrm{~S}$ & 48.5 & 55.6 & 52.4 & 35.6 & 43.8 & 45.5 & 49.6 & 37.0 & 57.6 & 44.9 \\
\hline \multirow[t]{3}{*}{ Ceftriaxone } & $\mathrm{MIC}_{50}$ & 32 & 16 & 16 & 64 & 32 & 32 & 32 & $\geq 128$ & 16 & 32 \\
\hline & $\mathrm{MIC}_{90}$ & $\geq 128$ & $\geq 128$ & $\geq 128$ & $\geq 128$ & $\geq 128$ & $\geq 128$ & $\geq 128$ & $\geq 128$ & $\geq 128$ & $\geq 128$ \\
\hline & $\% \mathrm{~S}$ & 30.1 & 30.6 & 35.8 & 19.3 & 25.8 & 22.1 & 27.0 & 20.4 & 31.6 & 25.9 \\
\hline \multirow[t]{3}{*}{ Levofloxacin } & $\mathrm{MIC}_{50}$ & 0.5 & 0.25 & 0.25 & 8 & 8 & 4 & 2 & 8 & 1 & 4 \\
\hline & $\mathrm{MIC}_{90}$ & $\geq 16$ & $\geq 16$ & $\geq 16$ & $\geq 16$ & $\geq 16$ & $\geq 16$ & $\geq 16$ & $\geq 16$ & $\geq 16$ & $\geq 16$ \\
\hline & $\% \mathrm{~S}$ & 55.3 & 62.5 & 56.9 & 33.1 & 40.5 & 46.8 & 51.1 & 35.8 & 53.7 & 44.4 \\
\hline \multirow[t]{3}{*}{ Minocycline } & $\mathrm{MIC}_{50}$ & $\leq 0.5$ & $\leq 0.5$ & $\leq 0.5$ & 1 & $\leq 0.5$ & 1 & 1 & 2 & $\leq 0.5$ & $\leq 0.5$ \\
\hline & $\mathrm{MIC}_{90}$ & 4 & 8 & 8 & 8 & 8 & 4 & 8 & 8 & 8 & 8 \\
\hline & $\% \mathrm{~S}$ & 91.3 & 80.6 & 87.3 & 80.5 & 88.2 & 97.4 & 81.5 & 68.5 & 88.7 & 84.1 \\
\hline \multirow[t]{3}{*}{ Pip-taz } & $\mathrm{MIC}_{50}$ & 16 & 8 & 4 & 128 & 16 & 8 & 16 & 64 & 16 & 16 \\
\hline & $\mathrm{MIC}_{90}$ & $\geq 256$ & $\geq 256$ & $\geq 256$ & $\geq 256$ & $\geq 256$ & $\geq 256$ & $\geq 256$ & $\geq 256$ & $\geq 256$ & $\geq 256$ \\
\hline & $\% \mathrm{~S}$ & 53.4 & 56.9 & 59.9 & 36.5 & 53.8 & 61.0 & 54.4 & 43.8 & 59.3 & 50.5 \\
\hline \multirow[t]{3}{*}{ Tigecycline } & $\mathrm{MIC}_{50}$ & 0.5 & 0.5 & 0.5 & 0.5 & 0.5 & 0.5 & 0.5 & 0.5 & 0.5 & 0.5 \\
\hline & $\mathrm{MIC}_{90}$ & 1 & 1 & 1 & 2 & 2 & 1 & 2 & 1 & 2 & 2 \\
\hline & $\% \mathrm{~S}$ & - & - & - & - & - & - & - & - & - & - \\
\hline $\begin{array}{l}\text { MDR Acinetobacter } \\
\text { baumannii }\end{array}$ & & $N=28$ & $N=23$ & $N=72$ & $N=326$ & $N=206$ & $N=13$ & $N=124$ & $N=50$ & $N=41$ & $N=883$ \\
\hline \multirow[t]{3}{*}{ Amikacin } & $\mathrm{MIC}_{50}$ & $\geq 128$ & $\geq 128$ & 64 & $\geq 128$ & 32 & 4 & 32 & $\geq 128$ & 64 & 64 \\
\hline & $\mathrm{MIC}_{90}$ & $\geq 128$ & $\geq 128$ & $\geq 128$ & $\geq 128$ & $\geq 128$ & 8 & $\geq 128$ & $\geq 128$ & $\geq 128$ & $\geq 128$ \\
\hline & $\% \mathrm{~S}$ & 10.7 & 8.7 & 36.1 & 16.0 & 42.2 & 100 & 40.3 & 6.0 & 12.2 & 27.3 \\
\hline \multirow[t]{3}{*}{ Carbapenems } & $\mathrm{MIC}_{50}$ & 16 & $\geq 32$ & $\geq 32$ & $\geq 32$ & $\geq 32$ & $\geq 32$ & $\geq 32$ & $\geq 32$ & $\geq 32$ & $\geq 32$ \\
\hline & $\mathrm{MIC}_{90}$ & $\geq 32$ & $\geq 32$ & $\geq 32$ & $\geq 32$ & $\geq 32$ & $\geq 32$ & $\geq 32$ & $\geq 32$ & $\geq 32$ & $\geq 32$ \\
\hline & $\% \mathrm{~S}$ & 21.4 & 8.7 & 15.3 & 4.6 & 12.1 & 0.0 & 1.6 & 10.0 & 26.8 & 8.7 \\
\hline \multirow[t]{3}{*}{ Cefepime } & $\mathrm{MIC}_{50}$ & $\geq 64$ & $\geq 64$ & $\geq 64$ & $\geq 64$ & $\geq 64$ & 32 & 32 & $\geq 64$ & 32 & $\geq 64$ \\
\hline & $\mathrm{MIC}_{90}$ & $\geq 64$ & $\geq 64$ & $\geq 64$ & $\geq 64$ & $\geq 64$ & $\geq 64$ & $\geq 64$ & $\geq 64$ & $\geq 64$ & $\geq 64$ \\
\hline & $\% \mathrm{~S}$ & 0.0 & 4.3 & 4.2 & 10.4 & 2.4 & 0.0 & 5.6 & 2.0 & 2.4 & 5.9 \\
\hline \multirow[t]{3}{*}{ Ceftazidime } & $\mathrm{MIC}_{50}$ & $\geq 64$ & $\geq 64$ & $\geq 64$ & $\geq 64$ & $\geq 64$ & $\geq 64$ & $\geq 64$ & $\geq 64$ & $\geq 64$ & $\geq 64$ \\
\hline & $\mathrm{MIC}_{90}$ & $\geq 64$ & $\geq 64$ & $\geq 64$ & $\geq 64$ & $\geq 64$ & $\geq 64$ & $\geq 64$ & $\geq 64$ & $\geq 64$ & $\geq 64$ \\
\hline & $\% \mathrm{~S}$ & 3.6 & 4.3 & 1.4 & 9.2 & 3.4 & 0.0 & 4.0 & 2.0 & 0.0 & 5.2 \\
\hline Ceftriaxone & $\mathrm{MIC}_{50}$ & $\geq 128$ & $\geq 128$ & $\geq 128$ & $\geq 128$ & $\geq 128$ & $\geq 128$ & $\geq 128$ & $\geq 128$ & $\geq 128$ & $\geq 128$ \\
\hline
\end{tabular}


Table 2 Antimicrobial susceptibility for Acinetobacter baumannii and multidrug-resistant (MDR) A. baumannii (Continued)

\begin{tabular}{|c|c|c|c|c|c|c|c|c|c|c|c|}
\hline & $\mathrm{MIC}_{90}$ & $\geq 128$ & $\geq 128$ & $\geq 128$ & $\geq 128$ & $\geq 128$ & $\geq 128$ & $\geq 128$ & $\geq 128$ & $\geq 128$ & $\geq 128$ \\
\hline & $\% \mathrm{~S}$ & 3.6 & 0.0 & 0.0 & 0.9 & 0.5 & 0.0 & 0.8 & 0.0 & 0.0 & 0.7 \\
\hline \multirow[t]{3}{*}{ Levofloxacin } & $\mathrm{MIC}_{50}$ & $\geq 16$ & 8 & 8 & $\geq 16$ & $\geq 16$ & $\geq 16$ & $\geq 16$ & $\geq 16$ & $\geq 16$ & $\geq 16$ \\
\hline & $\mathrm{MIC}_{90}$ & $\geq 16$ & $\geq 16$ & $\geq 16$ & $\geq 16$ & $\geq 16$ & $\geq 16$ & $\geq 16$ & $\geq 16$ & $\geq 16$ & $\geq 16$ \\
\hline & $\% \mathrm{~S}$ & 0.0 & 4.3 & 2.8 & 0.9 & 2.4 & 0.0 & 0.0 & 0.0 & 0.0 & 1.2 \\
\hline \multirow[t]{3}{*}{ Minocycline } & $\mathrm{MIC}_{50}$ & 1 & 4 & 4 & 2 & 2 & 2 & 4 & 4 & 4 & 2 \\
\hline & $\mathrm{MIC}_{90}$ & 8 & 8 & 16 & 8 & 16 & 4 & 8 & 8 & 8 & 8 \\
\hline & $\% \mathrm{~S}$ & 82.1 & 56.5 & 69.4 & 77.9 & 72.3 & 92.3 & 65.3 & 54.0 & 68.3 & 72.1 \\
\hline \multirow[t]{3}{*}{ Pip-taz } & $\mathrm{MIC}_{50}$ & $\geq 256$ & $\geq 256$ & $\geq 256$ & $\geq 256$ & 128 & 128 & $\geq 256$ & $\geq 256$ & $\geq 256$ & $\geq 256$ \\
\hline & $\mathrm{MIC}_{90}$ & $\geq 256$ & $\geq 256$ & $\geq 256$ & $\geq 256$ & $\geq 256$ & $\geq 256$ & $\geq 256$ & $\geq 256$ & $\geq 256$ & $\geq 256$ \\
\hline & $\% \mathrm{~S}$ & 3.6 & 4.3 & 8.3 & 3.1 & 10.7 & 15.4 & 3.2 & 2.0 & 2.4 & 5.4 \\
\hline \multirow[t]{3}{*}{ Tigecycline } & $\mathrm{MIC}_{50}$ & 1 & 1 & 1 & 1 & 1 & 1 & 1 & 1 & 1 & 1 \\
\hline & $\mathrm{MIC}_{90}$ & 2 & 1 & 2 & 2 & 2 & 4 & 2 & 2 & 2 & 2 \\
\hline & $\% \mathrm{~S}$ & - & - & - & - & - & - & - & - & - & - \\
\hline
\end{tabular}

Amoxicillin-clavulanate and ampicillin are not presented in this table as they are inactive against A. baumannii.

$\%$ S, \% susceptible, pip-taz piperacillin-tazobactam, carbapenems = imipenem $/$ meropenem.

- No CLSI approved interpretive criteria availableData not presented when $\mathrm{N}<10$ isolates.

and E. coli ATCC 25922 (ESBL-negative) were included for quality control.

\section{Statistical analyses}

The Fisher's Exact test (SAS, Version 8.2) was used to assess the relationships between the susceptibility/nonsusceptibility results presented in the current report compared with previous T.E.S.T. study data. Comparisons that yielded $\mathrm{p}$ values $\leq 0.01$ were considered significant.

\section{Results}

\section{Acinetobacter baumannii}

A total of 2900 isolates of A. baumannii were evaluated (Table 2). Regardless of the region, the $\mathrm{MIC}_{90}$ for tigecycline was $\leq 2 \mathrm{mg} / \mathrm{L}\left(\mathrm{MIC}_{50} 0.5 \mathrm{mg} / \mathrm{L}\right)$, but formal conclusion regarding susceptibility was not possible because breakpoint values have not been established. The isolates were also highly susceptible to minocycline (84.1\%). Carbapenem susceptibility ranged from $50 \%$ in East North Central to $80 \%$ in West South Central. In addition, some susceptibility patterns varied significantly among census regions. For example, there was dramatic regional variation in the numbers of isolates susceptible to amikacin in the East North Central (58.8\% susceptible) region compared to the numbers obtained from the New England (100\% susceptible) region.

A total of $883(30.4 \%)$ A. baumannii isolates were multidrug resistant (MDR, resistant to three or more classes of antimicrobial agent [ $\beta$-lactams, aminoglycosides, carbapenems or fluoroquinolones]) and the frequencies ranged from 16.9\% (13/77) in the New England region to
47.0\% (326/694) in the East North Central region. Furthermore, MDR isolates were susceptible to minocycline $\left(\mathrm{MIC}_{50} 2 \mathrm{mg} / \mathrm{L}, \mathrm{MIC}_{90} 8 \mathrm{mg} / \mathrm{L}\right)$ in some instances, but minocycline-nonsusceptible isolates were commonly recovered from the East South Central (54\% susceptibility) and Mountain regions (56.5\%) (Table 2). In contrast, MDR $A$. baumannii appeared universally susceptible to tigecycline $\left(\mathrm{MIC}_{50} 1 \mathrm{mg} / \mathrm{L}, \mathrm{MIC}_{90} 2 \mathrm{mg} / \mathrm{L}\right)$.

\section{Escherichia coli}

Non-ESBL-producing E. coli isolates $(\mathrm{n}=6643)$ were highly susceptible to amikacin, carbapenems, cefepime, ceftriaxone, and tigecycline (Table 3). In contrast, the organisms were significantly less susceptible to ampicillin, and the frequency of resistant organisms varied widely by region (e.g. susceptibility rates of $41.4 \%$ in East South Central region, $54.1 \%$ in the West North Central region). E. coli isolates that produced ESBL were also relatively uncommon (277 [4.0\%] of 6920 isolates), but the highest frequency was detected in isolates from the Mountain region $(11.1 \%, 26 / 235)$. In addition, ESBL producers were highly susceptible to amikacin $\left(\mathrm{MIC}_{50} 4 \mathrm{mg} / \mathrm{L}\right.$, $\mathrm{MIC}_{90} 16 \mathrm{mg} / \mathrm{L}, 94.9 \%$ susceptible), carbapenems $\left(\mathrm{MIC}_{50} \leq\right.$ $0.06 \mathrm{mg} / \mathrm{L}, \mathrm{MIC}_{90} 0.25 \mathrm{mg} / \mathrm{L}, 98.2 \%$ susceptible), and tigecycline $\left(\mathrm{MIC}_{50} 0.25 \mathrm{mg} / \mathrm{L}, \mathrm{MIC}_{90} 0.5 \mathrm{mg} / \mathrm{L}, 100 \%\right.$ susceptible) (Table 4).

\section{Klebsiella pneumoniae and $K$. oxytoca}

Non-ESBL producing $K$. pneumoniae and $K$. oxytoca (Table 3$)$ were highly susceptible $(>90 \%)$ to cefepime, carbapenems, amikacin, and tigecycline regardless of region; susceptibility was only slightly lower for 
Table 3 Antimicrobial susceptibility for Enterobacteriaceae

\begin{tabular}{|c|c|c|c|c|c|c|c|c|c|c|c|}
\hline & & Pacific & Mountain & $\begin{array}{c}\text { West North } \\
\text { Central }\end{array}$ & $\begin{array}{c}\text { East North } \\
\text { Central }\end{array}$ & $\begin{array}{c}\text { Middle } \\
\text { Atlantic }\end{array}$ & $\begin{array}{c}\text { New } \\
\text { England }\end{array}$ & $\begin{array}{c}\text { South } \\
\text { Atlantic }\end{array}$ & $\begin{array}{c}\text { East South } \\
\text { Central }\end{array}$ & $\begin{array}{c}\text { West South } \\
\text { Central }\end{array}$ & USA \\
\hline $\begin{array}{l}\text { E. coli } \\
\text { (ESBL negative) }\end{array}$ & & $N=230$ & $N=209$ & $N=801$ & $N=1411$ & $N=1811$ & $N=220$ & $N=1192$ & $N=384$ & $N=385$ & $N=6643$ \\
\hline \multirow[t]{3}{*}{ Amikacin } & $\mathrm{MIC}_{50}$ & 2 & 2 & 2 & 2 & 2 & 2 & 2 & 2 & 2 & 2 \\
\hline & $\mathrm{MIC}_{90}$ & 4 & 8 & 4 & 4 & 4 & 4 & 4 & 8 & 4 & 4 \\
\hline & $\% \mathrm{~S}$ & 99.6 & 99.0 & 100 & 99.5 & 99.3 & 99.5 & 99.0 & 98.2 & 99.7 & 99.4 \\
\hline \multirow[t]{3}{*}{ Amoxi-clav } & $\mathrm{MIC}_{50}$ & 4 & 8 & 4 & 8 & 8 & 4 & 4 & 8 & 8 & 8 \\
\hline & $\mathrm{MIC}_{90}$ & 16 & 32 & 16 & 16 & 16 & 32 & 32 & 32 & 16 & 16 \\
\hline & $\% \mathrm{~S}$ & 77.0 & 71.8 & 80.8 & 75.7 & 76.1 & 76.4 & 75.4 & 70.1 & 76.4 & 76.0 \\
\hline \multirow[t]{3}{*}{ Ampicillin } & $\mathrm{MIC}_{50}$ & 4 & $\geq 64$ & 4 & 32 & $\geq 64$ & 8 & $\geq 64$ & $\geq 64$ & $\geq 64$ & 32 \\
\hline & $\mathrm{MIC}_{90}$ & $\geq 64$ & $\geq 64$ & $\geq 64$ & $\geq 64$ & $\geq 64$ & $\geq 64$ & $\geq 64$ & $\geq 64$ & $\geq 64$ & $\geq 64$ \\
\hline & $\% \mathrm{~S}$ & 52.6 & 44.0 & 54.1 & 47.4 & 46.3 & 51.4 & 45.4 & 41.4 & 43.4 & 47.2 \\
\hline \multirow[t]{3}{*}{ Carbapenems } & $\mathrm{MIC}_{50}$ & $\leq 0.06$ & $\leq 0.06$ & $\leq 0.06$ & $\leq 0.06$ & $\leq 0.06$ & $\leq 0.06$ & $\leq 0.06$ & $\leq 0.06$ & $\leq 0.06$ & $\leq 0.06$ \\
\hline & $\mathrm{MIC}_{90}$ & 0.25 & 0.25 & 0.25 & 0.12 & 0.25 & 0.25 & 0.25 & 0.25 & 0.25 & 0.25 \\
\hline & $\% \mathrm{~S}$ & 100 & 99.0 & 99.6 & 99.7 & 99.6 & 99.1 & 99.1 & 97.7 & 99.7 & 99.4 \\
\hline \multirow[t]{3}{*}{ Cefepime } & $\mathrm{MIC}_{50}$ & $\leq 0.5$ & $\leq 0.5$ & $\leq 0.5$ & $\leq 0.5$ & $\leq 0.5$ & $\leq 0.5$ & $\leq 0.5$ & $\leq 0.5$ & $\leq 0.5$ & $\leq 0.5$ \\
\hline & $\mathrm{MIC}_{90}$ & $\leq 0.5$ & $\leq 0.5$ & $\leq 0.5$ & $\leq 0.5$ & $\leq 0.5$ & $\leq 0.5$ & $\leq 0.5$ & $\leq 0.5$ & $\leq 0.5$ & $\leq 0.5$ \\
\hline & $\% \mathrm{~S}$ & 99.1 & 99.0 & 99.6 & 99.1 & 98.8 & 99.1 & 98.5 & 98.2 & 99.5 & 99.0 \\
\hline \multirow[t]{3}{*}{ Ceftriaxone } & $\mathrm{MIC}_{50}$ & $\leq 0.06$ & $\leq 0.06$ & $\leq 0.06$ & $\leq 0.06$ & $\leq 0.06$ & $\leq 0.06$ & $\leq 0.06$ & $\leq 0.06$ & $\leq 0.06$ & $\leq 0.06$ \\
\hline & $\mathrm{MIC}_{90}$ & 0.12 & 1 & 0.12 & 0.25 & 0.25 & 0.25 & 0.25 & 0.5 & 0.12 & 0.25 \\
\hline & $\% \mathrm{~S}$ & 97.0 & 90.4 & 96.9 & 96.7 & 93.9 & 93.2 & 94.3 & 91.1 & 96.4 & 94.9 \\
\hline \multirow[t]{3}{*}{ Levofloxacin } & $\mathrm{MIC}_{50}$ & 0.03 & 0.03 & 0.03 & 0.03 & 0.03 & 0.03 & 0.03 & 0.03 & 0.03 & 0.03 \\
\hline & $\mathrm{MIC}_{90}$ & $\geq 16$ & $\geq 16$ & $\geq 16$ & $\geq 16$ & $\geq 16$ & $\geq 16$ & $\geq 16$ & $\geq 16$ & $\geq 16$ & $\geq 16$ \\
\hline & $\% \mathrm{~S}$ & 77.8 & 76.1 & 79.7 & 74.7 & 72.5 & 78.6 & 70.6 & 72.1 & 74.0 & 74.0 \\
\hline \multirow[t]{3}{*}{ Minocycline } & $\mathrm{MIC}_{50}$ & 1 & 1 & 1 & 1 & 1 & 1 & 1 & 1 & 1 & 1 \\
\hline & $\mathrm{MIC}_{90}$ & 8 & 16 & 8 & 8 & 8 & 8 & 8 & 8 & 8 & 8 \\
\hline & $\% \mathrm{~S}$ & 86.1 & 80.4 & 89.6 & 84.5 & 83.4 & 83.6 & 85.2 & 85.9 & 86.0 & 85.0 \\
\hline \multirow[t]{3}{*}{ Pip-taz } & $\mathrm{MIC}_{50}$ & 1 & 1 & 1 & 1 & 1 & 1 & 1 & 1 & 1 & 1 \\
\hline & $\mathrm{MIC}_{90}$ & 4 & 4 & 4 & 4 & 4 & 4 & 4 & 4 & 4 & 4 \\
\hline & $\% \mathrm{~S}$ & 95.2 & 94.3 & 97.5 & 96.7 & 97.1 & 96.8 & 96.1 & 96.9 & 97.7 & 96.7 \\
\hline \multirow[t]{3}{*}{ Tigecycline } & $\mathrm{MIC}_{50}$ & 0.12 & 0.12 & 0.12 & 0.12 & 0.12 & 0.12 & 0.12 & 0.12 & 0.12 & 0.12 \\
\hline & $\mathrm{MIC}_{90}$ & 0.25 & 0.25 & 0.25 & 0.5 & 0.5 & 0.25 & 0.25 & 0.25 & 1 & 0.5 \\
\hline & $\% \mathrm{~S}$ & 100 & 100 & 99.9 & 100 & 99.9 & 100 & 100 & 100 & 100 & 100 \\
\hline $\begin{array}{l}\text { K. pneumoniae } \\
\text { (ESBL negative) }\end{array}$ & & $N=159$ & $N=151$ & $N=575$ & $N=1061$ & $N=1250$ & $N=150$ & $N=991$ & $N=320$ & $N=294$ & $N=4951$ \\
\hline \multirow[t]{3}{*}{ Amikacin } & $\mathrm{MIC}_{50}$ & 2 & 1 & 1 & 1 & 2 & 1 & 2 & 1 & 1 & 1 \\
\hline & $\mathrm{MIC}_{90}$ & 2 & 2 & 2 & 2 & 8 & 2 & 2 & 2 & 2 & 2 \\
\hline & $\% \mathrm{~S}$ & 100 & 98.7 & 99.5 & 99.8 & 97.2 & 99.3 & 98.6 & 99.1 & 99.0 & 98.7 \\
\hline \multirow[t]{3}{*}{ Amoxi-clav } & $\mathrm{MIC}_{50}$ & 2 & 2 & 2 & 2 & 2 & 2 & 2 & 2 & 2 & 2 \\
\hline & $\mathrm{MIC}_{90}$ & 8 & 16 & 8 & 8 & 32 & 8 & 16 & 16 & 8 & 16 \\
\hline & $\% \mathrm{~S}$ & 95.0 & 88.7 & 93.6 & 90.8 & 82.2 & 90.0 & 87.4 & 89.1 & 91.2 & 88.2 \\
\hline \multirow[t]{3}{*}{ Ampicillin } & $\mathrm{MIC}_{50}$ & 32 & $\geq 64$ & 32 & $\geq 64$ & $\geq 64$ & $\geq 64$ & $\geq 64$ & $\geq 64$ & 32 & $\geq 64$ \\
\hline & $\mathrm{MIC}_{90}$ & $\geq 64$ & $\geq 64$ & $\geq 64$ & $\geq 64$ & $\geq 64$ & $\geq 64$ & $\geq 64$ & $\geq 64$ & $\geq 64$ & $\geq 64$ \\
\hline & $\% \mathrm{~S}$ & 2.5 & 0.7 & 1.9 & 1.2 & 2.6 & 4.0 & 1.7 & 1.3 & 1.7 & 1.9 \\
\hline Carbapenems & $\mathrm{MIC}_{50}$ & $\leq 0.06$ & $\leq 0.06$ & $\leq 0.06$ & $\leq 0.06$ & $\leq 0.06$ & $\leq 0.06$ & $\leq 0.06$ & $\leq 0.06$ & $\leq 0.06$ & $\leq 0.06$ \\
\hline
\end{tabular}


Table 3 Antimicrobial susceptibility for Enterobacteriaceae (Continued)

\begin{tabular}{|c|c|c|c|c|c|c|c|c|c|c|c|}
\hline & $\mathrm{MIC}_{90}$ & 0.12 & 0.5 & 0.25 & 0.25 & 0.5 & 0.12 & 0.25 & 0.12 & 0.5 & 0.25 \\
\hline & $\% \mathrm{~S}$ & 100 & 97.4 & 99.5 & 98.8 & 92.5 & 99.3 & 97.8 & 99.1 & 99.7 & 97.2 \\
\hline \multirow[t]{3}{*}{ Cefepime } & $\mathrm{MIC}_{50}$ & $\leq 0.5$ & $\leq 0.5$ & $\leq 0.5$ & $\leq 0.5$ & $\leq 0.5$ & $\leq 0.5$ & $\leq 0.5$ & $\leq 0.5$ & $\leq 0.5$ & $\leq 0.5$ \\
\hline & $\mathrm{MIC}_{90}$ & $\leq 0.5$ & $\leq 0.5$ & $\leq 0.5$ & $\leq 0.5$ & 4 & $\leq 0.5$ & $\leq 0.5$ & $\leq 0.5$ & $\leq 0.5$ & $\leq 0.5$ \\
\hline & $\% \mathrm{~S}$ & 99.4 & 98.0 & 99.1 & 99.4 & 93.0 & 99.3 & 98.2 & 99.1 & 99.0 & 97.4 \\
\hline \multirow[t]{3}{*}{ Ceftriaxone } & $\mathrm{MIC}_{50}$ & $\leq 0.06$ & $\leq 0.06$ & $\leq 0.06$ & $\leq 0.06$ & $\leq 0.06$ & $\leq 0.06$ & $\leq 0.06$ & $\leq 0.06$ & $\leq 0.06$ & $\leq 0.06$ \\
\hline & $\mathrm{MIC}_{90}$ & 0.12 & 0.5 & 0.25 & 0.25 & 8 & 0.25 & 0.5 & 0.25 & 0.25 & 0.5 \\
\hline & $\% \mathrm{~S}$ & 98.7 & 91.4 & 96.5 & 95.4 & 84.6 & 94.0 & 91.7 & 96.3 & 97.3 & 92.2 \\
\hline \multirow[t]{3}{*}{ Levofloxacin } & $\mathrm{MIC}_{50}$ & 0.06 & 0.06 & 0.06 & 0.06 & 0.06 & 0.06 & 0.06 & 0.06 & 0.06 & 0.06 \\
\hline & $\mathrm{MIC}_{90}$ & 0.25 & 0.5 & 0.5 & 0.5 & $\geq 16$ & 1 & 1 & 1 & 0.5 & 1 \\
\hline & $\% \mathrm{~S}$ & 99.4 & 93.4 & 96.3 & 95.5 & 86.2 & 90.7 & 92.9 & 94.7 & 97.3 & 92.7 \\
\hline \multirow[t]{3}{*}{ Minocycline } & $\mathrm{MIC}_{50}$ & 2 & 2 & 2 & 2 & 2 & 2 & 2 & 2 & 2 & 2 \\
\hline & $\mathrm{MIC}_{90}$ & 16 & 16 & 8 & 16 & 16 & 8 & 16 & 16 & 8 & 16 \\
\hline & $\% \mathrm{~S}$ & 84.9 & 78.8 & 85.2 & 79.5 & 80.4 & 85.3 & 82.0 & 82.2 & 83.3 & 81.6 \\
\hline \multirow[t]{3}{*}{ Pip-taz } & $\mathrm{MIC}_{50}$ & 2 & 2 & 2 & 2 & 2 & 2 & 2 & 2 & 2 & 2 \\
\hline & $\mathrm{MIC}_{90}$ & 8 & 8 & 8 & 8 & 64 & 4 & 8 & 8 & 8 & 8 \\
\hline & $\% \mathrm{~S}$ & 96.9 & 92.1 & 97.6 & 96.0 & 88.6 & 95.3 & 94.6 & 97.8 & 96.6 & 94.1 \\
\hline \multirow[t]{3}{*}{ Tigecycline } & $\mathrm{MIC}_{50}$ & 0.5 & 0.5 & 0.5 & 0.5 & 0.5 & 0.5 & 0.5 & 0.5 & 0.5 & 0.5 \\
\hline & $\mathrm{MIC}_{90}$ & 1 & 1 & 1 & 1 & 2 & 1 & 2 & 2 & 2 & 2 \\
\hline & $\% \mathrm{~S}$ & 96.2 & 94.7 & 95.5 & 96.4 & 94.9 & 97.3 & 95.9 & 96.6 & 96.3 & 95.8 \\
\hline Klebsiella oxytoca & & $N=57$ & $N=50$ & $N=176$ & $N=301$ & $N=279$ & $N=45$ & $N=145$ & $N=49$ & $N=68$ & $N=1170$ \\
\hline \multirow[t]{3}{*}{ Amikacin } & $\mathrm{MIC}_{50}$ & 2 & 2 & 2 & 2 & 2 & 1 & 2 & 2 & 1 & 2 \\
\hline & $\mathrm{MIC}_{90}$ & 4 & 4 & 4 & 4 & 4 & 2 & 4 & 4 & 2 & 4 \\
\hline & $\% \mathrm{~S}$ & 100 & 96.0 & 100 & 99.7 & 98.9 & 100 & 97.2 & 100 & 100 & 99.1 \\
\hline \multirow[t]{3}{*}{ Amoxi-clav } & $\mathrm{MIC}_{50}$ & 2 & 2 & 4 & 2 & 4 & 2 & 2 & 2 & 4 & 2 \\
\hline & $\mathrm{MIC}_{90}$ & 8 & 32 & 32 & 16 & 32 & 4 & 32 & 8 & 8 & 16 \\
\hline & $\% \mathrm{~S}$ & 93.0 & 82.0 & 82.4 & 88.7 & 79.6 & 97.8 & 84.8 & 91.8 & 91.2 & 85.6 \\
\hline \multirow[t]{3}{*}{ Ampicillin } & $\mathrm{MIC}_{50}$ & $\geq 64$ & $\geq 64$ & $\geq 64$ & $\geq 64$ & $\geq 64$ & 32 & $\geq 64$ & $\geq 64$ & $\geq 64$ & $\geq 64$ \\
\hline & $\mathrm{MIC}_{90}$ & $\geq 64$ & $\geq 64$ & $\geq 64$ & $\geq 64$ & $\geq 64$ & $\geq 64$ & $\geq 64$ & $\geq 64$ & $\geq 64$ & $\geq 64$ \\
\hline & $\% \mathrm{~S}$ & 0.0 & 2.0 & 0.6 & 2.3 & 0.0 & 2.2 & 2.1 & 0.0 & 0.0 & 1.1 \\
\hline \multirow[t]{3}{*}{ Carbapenems } & $\mathrm{MIC}_{50}$ & $\leq 0.06$ & $\leq 0.06$ & $\leq 0.06$ & $\leq 0.06$ & $\leq 0.06$ & $\leq 0.06$ & $\leq 0.06$ & $\leq 0.06$ & $\leq 0.06$ & $\leq 0.06$ \\
\hline & $\mathrm{MIC}_{90}$ & 0.12 & 0.5 & 0.25 & 0.25 & 0.5 & 0.25 & 0.25 & 0.12 & 0.25 & 0.25 \\
\hline & $\% \mathrm{~S}$ & 100 & 94.0 & 100 & 98.7 & 99.6 & 100 & 97.9 & 98.0 & 100 & 99.0 \\
\hline \multirow[t]{3}{*}{ Cefepime } & $\mathrm{MIC}_{50}$ & $\leq 0.5$ & $\leq 0.5$ & $\leq 0.5$ & $\leq 0.5$ & $\leq 0.5$ & $\leq 0.5$ & $\leq 0.5$ & $\leq 0.5$ & $\leq 0.5$ & $\leq 0.5$ \\
\hline & $\mathrm{MIC}_{90}$ & $\leq 0.5$ & 2 & 2 & 1 & 2 & $\leq 0.5$ & 1 & $\leq 0.5$ & $\leq 0.5$ & 1 \\
\hline & $\% \mathrm{~S}$ & 100 & 96.0 & 98.9 & 98.7 & 98.6 & 100 & 96.6 & 100 & 100 & 98.5 \\
\hline \multirow[t]{3}{*}{ Ceftriaxone } & $\mathrm{MIC}_{50}$ & $\leq 0.06$ & $\leq 0.06$ & $\leq 0.06$ & $\leq 0.06$ & $\leq 0.06$ & $\leq 0.06$ & $\leq 0.06$ & $\leq 0.06$ & $\leq 0.06$ & $\leq 0.06$ \\
\hline & $\mathrm{MIC}_{90}$ & 0.25 & 4 & 4 & 2 & 16 & 0.25 & 4 & 0.5 & 0.5 & 4 \\
\hline & $\% \mathrm{~S}$ & 93.0 & 86.0 & 83.0 & 88.7 & 77.8 & 97.8 & 85.5 & 91.8 & 92.6 & 85.6 \\
\hline \multirow[t]{3}{*}{ Levofloxacin } & $\mathrm{MIC}_{50}$ & 0.03 & 0.03 & 0.06 & 0.06 & 0.06 & 0.03 & 0.03 & 0.03 & 0.06 & 0.06 \\
\hline & $\mathrm{MIC}_{90}$ & 0.5 & 0.12 & 2 & 0.25 & 2 & 0.06 & 0.5 & 0.5 & 0.5 & 0.5 \\
\hline & $\% \mathrm{~S}$ & 94.7 & 96.0 & 90.9 & 95.0 & 93.2 & 100 & 93.1 & 95.9 & 95.6 & 94.0 \\
\hline \multirow[t]{3}{*}{ Minocycline } & $\mathrm{MIC}_{50}$ & 1 & 1 & 1 & 2 & 2 & 1 & 1 & 1 & 1 & 1 \\
\hline & $\mathrm{MIC}_{90}$ & 2 & 16 & 4 & 8 & 8 & 2 & 4 & 4 & 8 & 8 \\
\hline & $\% \mathrm{~S}$ & 98.2 & 86.0 & 90.3 & 89.0 & 87.1 & 95.6 & 91.0 & 95.9 & 85.3 & 89.7 \\
\hline
\end{tabular}


Table 3 Antimicrobial susceptibility for Enterobacteriaceae (Continued)

\begin{tabular}{|c|c|c|c|c|c|c|c|c|c|c|c|}
\hline \multirow[t]{3}{*}{ Pip-taz } & $\mathrm{MIC}_{50}$ & 2 & 1 & 1 & 1 & 1 & 1 & 1 & 1 & 2 & 1 \\
\hline & $\mathrm{MIC}_{90}$ & 4 & 32 & $\geq 256$ & 8 & $\geq 256$ & 2 & 8 & 2 & 8 & 16 \\
\hline & $\% \mathrm{~S}$ & 94.7 & 88.0 & 86.9 & 92.4 & 86.0 & 97.8 & 91.7 & 98.0 & 94.1 & 90.4 \\
\hline \multirow[t]{3}{*}{ Tigecycline } & $\mathrm{MIC}_{50}$ & 0.25 & 0.25 & 0.25 & 0.25 & 0.5 & 0.25 & 0.25 & 0.25 & 0.5 & 0.25 \\
\hline & $\mathrm{MIC}_{90}$ & 0.5 & 1 & 1 & 1 & 2 & 0.5 & 1 & 0.5 & 2 & 1 \\
\hline & $\% \mathrm{~S}$ & 100 & 98.0 & 99.4 & 99.0 & 98.2 & 100 & 97.9 & 100 & 100 & 98.9 \\
\hline Serratia marcescens & & $N=87$ & $N=61$ & $N=302$ & $N=544$ & $N=609$ & $N=67$ & $N=475$ & $N=140$ & $N=136$ & $N=2421$ \\
\hline \multirow[t]{3}{*}{ Amikacin } & $\mathrm{MIC}_{50}$ & 2 & 2 & 2 & 2 & 2 & 2 & 2 & 2 & 2 & 2 \\
\hline & $\mathrm{MIC}_{90}$ & 8 & 8 & 4 & 4 & 4 & 4 & 4 & 4 & 4 & 4 \\
\hline & $\% \mathrm{~S}$ & 98.9 & 98.4 & 99.3 & 99.3 & 99.2 & 100 & 98.9 & 97.9 & 99.3 & 99.1 \\
\hline \multirow[t]{3}{*}{ Amoxi-clav } & $\mathrm{MIC}_{50}$ & $\geq 64$ & $\geq 64$ & $\geq 64$ & $\geq 64$ & $\geq 64$ & $\geq 64$ & $\geq 64$ & $\geq 64$ & $\geq 64$ & $\geq 64$ \\
\hline & $\mathrm{MIC}_{90}$ & $\geq 64$ & $\geq 64$ & $\geq 64$ & $\geq 64$ & $\geq 64$ & $\geq 64$ & $\geq 64$ & $\geq 64$ & $\geq 64$ & $\geq 64$ \\
\hline & $\% \mathrm{~S}$ & 2.3 & 6.6 & 4.6 & 1.3 & 3.3 & 3.0 & 3.6 & 2.1 & 1.5 & 2.9 \\
\hline \multirow[t]{3}{*}{ Ampicillin } & $\mathrm{MIC}_{50}$ & $\geq 64$ & $\geq 64$ & $\geq 64$ & $\geq 64$ & $\geq 64$ & $\geq 64$ & $\geq 64$ & $\geq 64$ & $\geq 64$ & $\geq 64$ \\
\hline & $\mathrm{MIC}_{90}$ & $\geq 64$ & $\geq 64$ & $\geq 64$ & $\geq 64$ & $\geq 64$ & $\geq 64$ & $\geq 64$ & $\geq 64$ & $\geq 64$ & $\geq 64$ \\
\hline & $\% \mathrm{~S}$ & 2.3 & 3.3 & 3.3 & 1.3 & 1.3 & 3.0 & 1.1 & 0.7 & 0.7 & 1.6 \\
\hline \multirow[t]{3}{*}{ Carbapenems } & $\mathrm{MIC}_{50}$ & 0.12 & 0.12 & 0.12 & $\leq 0.06$ & 0.12 & 0.12 & 0.12 & 0.12 & $\leq 0.06$ & 0.12 \\
\hline & $\mathrm{MIC}_{90}$ & 1 & 1 & 1 & 0.5 & 1 & 0.12 & 0.5 & 0.25 & 0.5 & 1 \\
\hline & $\% \mathrm{~S}$ & 97.7 & 95.1 & 95.7 & 96.7 & 96.4 & 100 & 96.2 & 96.4 & 100 & 96.7 \\
\hline \multirow[t]{3}{*}{ Cefepime } & $\mathrm{MIC}_{50}$ & $\leq 0.5$ & $\leq 0.5$ & $\leq 0.5$ & $\leq 0.5$ & $\leq 0.5$ & $\leq 0.5$ & $\leq 0.5$ & $\leq 0.5$ & $\leq 0.5$ & $\leq 0.5$ \\
\hline & $\mathrm{MIC}_{90}$ & $\leq 0.5$ & 1 & 1 & 4 & 4 & $\leq 0.5$ & 1 & $\leq 0.5$ & 1 & 2 \\
\hline & $\% \mathrm{~S}$ & 100 & 100 & 98.0 & 95.4 & 97.2 & 100 & 97.7 & 97.9 & 100 & 97.4 \\
\hline \multirow[t]{3}{*}{ Ceftriaxone } & $\mathrm{MIC}_{50}$ & 0.25 & 0.25 & 0.25 & 0.25 & 0.25 & 0.25 & 0.25 & 0.25 & 0.25 & 0.25 \\
\hline & $\mathrm{MIC}_{90}$ & 2 & 2 & 4 & 32 & 16 & 1 & 4 & 4 & 2 & 8 \\
\hline & $\% \mathrm{~S}$ & 88.5 & 86.9 & 88.7 & 78.3 & 77.8 & 92.5 & 83.6 & 86.4 & 89.7 & 82.6 \\
\hline \multirow[t]{3}{*}{ Levofloxacin } & $\mathrm{MIC}_{50}$ & 0.12 & 0.12 & 0.12 & 0.12 & 0.12 & 0.12 & 0.12 & 0.12 & 0.12 & 0.12 \\
\hline & $\mathrm{MIC}_{90}$ & 1 & 0.5 & 1 & 2 & 2 & 0.5 & 1 & 1 & 0.5 & 1 \\
\hline & $\% \mathrm{~S}$ & 96.6 & 100 & 95.0 & 93.9 & 93.3 & 98.5 & 92.6 & 96.4 & 98.5 & 94.4 \\
\hline \multirow[t]{3}{*}{ Minocycline } & $\mathrm{MIC}_{50}$ & 2 & 4 & 4 & 4 & 4 & 2 & 4 & 4 & 4 & 4 \\
\hline & $\mathrm{MIC}_{90}$ & 8 & 8 & 8 & 8 & 8 & 8 & 8 & 8 & 8 & 8 \\
\hline & $\% \mathrm{~S}$ & 86.2 & 77.0 & 75.8 & 74.1 & 81.0 & 88.1 & 78.9 & 89.3 & 73.5 & 78.7 \\
\hline \multirow[t]{3}{*}{ Pip-taz } & $\mathrm{MIC}_{50}$ & 2 & 1 & 1 & 2 & 1 & 1 & 1 & 1 & 2 & 1 \\
\hline & $\mathrm{MIC}_{90}$ & 8 & 8 & 4 & 8 & 8 & 4 & 4 & 4 & 4 & 8 \\
\hline & $\% \mathrm{~S}$ & 98.9 & 96.7 & 97.0 & 93.6 & 94.1 & 98.5 & 96.2 & 97.1 & 97.8 & 95.5 \\
\hline \multirow[t]{3}{*}{ Tigecycline } & $\mathrm{MIC}_{50}$ & 1 & 1 & 1 & 1 & 1 & 1 & 1 & 1 & 1 & 1 \\
\hline & $\mathrm{MIC}_{90}$ & 2 & 2 & 2 & 2 & 2 & 2 & 2 & 1 & 2 & 2 \\
\hline & $\% \mathrm{~S}$ & 98.9 & 91.8 & 97.4 & 96.5 & 95.4 & 97.0 & 95.4 & 97.9 & 91.9 & 95.9 \\
\hline Enterobacter spp. & & $N=227$ & $N=192$ & $N=770$ & $N=1351$ & $N=1500$ & $N=198$ & $N=1118$ & $N=339$ & $N=370$ & $N=6065$ \\
\hline \multirow[t]{3}{*}{ Amikacin } & $\mathrm{MIC}_{50}$ & 2 & 2 & 2 & 2 & 2 & 2 & 2 & 2 & 2 & 2 \\
\hline & $\mathrm{MIC}_{90}$ & 4 & 4 & 2 & 4 & 4 & 2 & 4 & 2 & 4 & 4 \\
\hline & $\% \mathrm{~S}$ & 100 & 99.5 & 99.5 & 98.7 & 98.1 & 99.5 & 99.2 & 98.8 & 98.6 & 98.9 \\
\hline \multirow[t]{3}{*}{ Amoxi-clav } & $\mathrm{MIC}_{50}$ & $\geq 64$ & $\geq 64$ & $\geq 64$ & $\geq 64$ & $\geq 64$ & $\geq 64$ & $\geq 64$ & $\geq 64$ & $\geq 64$ & $\geq 64$ \\
\hline & $\mathrm{MIC}_{90}$ & $\geq 64$ & $\geq 64$ & $\geq 64$ & $\geq 64$ & $\geq 64$ & $\geq 64$ & $\geq 64$ & $\geq 64$ & $\geq 64$ & $\geq 64$ \\
\hline & $\% \mathrm{~S}$ & 4.8 & 4.7 & 7.3 & 3.3 & 3.7 & 2.5 & 5.5 & 6.2 & 5.1 & 4.7 \\
\hline Ampicillin & $\mathrm{MIC}_{50}$ & $\geq 64$ & $\geq 64$ & $\geq 64$ & $\geq 64$ & $\geq 64$ & $\geq 64$ & $\geq 64$ & $\geq 64$ & $\geq 64$ & $\geq 64$ \\
\hline
\end{tabular}


Table 3 Antimicrobial susceptibility for Enterobacteriaceae (Continued)

\begin{tabular}{|c|c|c|c|c|c|c|c|c|c|c|c|}
\hline & $\mathrm{MIC}_{90}$ & $\geq 64$ & $\geq 64$ & $\geq 64$ & $\geq 64$ & $\geq 64$ & $\geq 64$ & $\geq 64$ & $\geq 64$ & $\geq 64$ & $\geq 64$ \\
\hline & $\% \mathrm{~S}$ & 2.2 & 6.3 & 5.8 & 2.7 & 2.9 & 3.5 & 2.8 & 2.9 & 3.0 & 3.3 \\
\hline \multirow[t]{3}{*}{ Carbapenems } & $\mathrm{MIC}_{50}$ & $\leq 0.06$ & $\leq 0.06$ & $\leq 0.06$ & $\leq 0.06$ & 0.12 & $\leq 0.06$ & $\leq 0.06$ & $\leq 0.06$ & $\leq 0.06$ & $\leq 0.06$ \\
\hline & $\mathrm{MIC}_{90}$ & 0.5 & 1 & 0.5 & 0.5 & 1 & 0.5 & 0.5 & 0.5 & 0.5 & 0.5 \\
\hline & $\% \mathrm{~S}$ & 100 & 97.4 & 98.6 & 97.9 & 96.1 & 99.5 & 97.9 & 97.3 & 97.8 & 97.6 \\
\hline \multirow[t]{3}{*}{ Cefepime } & $\mathrm{MIC}_{50}$ & $\leq 0.5$ & $\leq 0.5$ & $\leq 0.5$ & $\leq 0.5$ & $\leq 0.5$ & $\leq 0.5$ & $\leq 0.5$ & $\leq 0.5$ & $\leq 0.5$ & $\leq 0.5$ \\
\hline & $\mathrm{MIC}_{90}$ & 2 & 4 & 2 & 4 & 4 & 2 & 4 & 4 & 2 & 4 \\
\hline & $\% \mathrm{~S}$ & 95.6 & 96.9 & 97.0 & 97.1 & 95.6 & 97.5 & 94.7 & 94.4 & 97.6 & 96.1 \\
\hline \multirow[t]{3}{*}{ Ceftriaxone } & $\mathrm{MIC}_{50}$ & 0.25 & 0.5 & 0.25 & 0.25 & 0.25 & 0.25 & 0.25 & 0.25 & 0.25 & 0.25 \\
\hline & $\mathrm{MIC}_{90}$ & 16 & 64 & 32 & 32 & 64 & 32 & 64 & 64 & 32 & 64 \\
\hline & $\% \mathrm{~S}$ & 79.7 & 63.5 & 76.9 & 72.8 & 63.8 & 74.7 & 71.7 & 64.3 & 76.8 & 70.7 \\
\hline \multirow[t]{3}{*}{ Levofloxacin } & $\mathrm{MIC}_{50}$ & 0.03 & 0.06 & 0.06 & 0.06 & 0.06 & 0.03 & 0.06 & 0.06 & 0.06 & 0.06 \\
\hline & $\mathrm{MIC}_{90}$ & 0.25 & 0.5 & 0.5 & 1 & 4 & 0.5 & 4 & $\geq 16$ & 0.5 & 2 \\
\hline & $\% \mathrm{~S}$ & 97.8 & 93.2 & 93.9 & 93.0 & 89.4 & 98.0 & 86.8 & 85.0 & 93.5 & 91.0 \\
\hline \multirow[t]{3}{*}{ Minocycline } & $\mathrm{MIC}_{50}$ & 2 & 2 & 2 & 4 & 2 & 2 & 2 & 2 & 2 & 2 \\
\hline & $\mathrm{MIC}_{90}$ & 8 & 16 & 16 & 16 & 16 & 4 & 8 & 8 & 16 & 16 \\
\hline & $\% \mathrm{~S}$ & 87.2 & 78.1 & 79.7 & 77.1 & 76.3 & 90.4 & 80.8 & 81.1 & 77.6 & 79.0 \\
\hline \multirow[t]{3}{*}{ Pip/taz } & $\mathrm{MIC}_{50}$ & 2 & 2 & 2 & 2 & 2 & 2 & 2 & 2 & 2 & 2 \\
\hline & $\mathrm{MIC}_{90}$ & 64 & 128 & 64 & 64 & 64 & 64 & 64 & 64 & 64 & 64 \\
\hline & $\% \mathrm{~S}$ & 85.0 & 80.2 & 86.5 & 84.3 & 80.9 & 85.4 & 82.0 & 80.2 & 83.2 & 83.0 \\
\hline \multirow[t]{3}{*}{ Tigecycline } & $\mathrm{MIC}_{50}$ & 0.5 & 0.5 & 0.5 & 0.5 & 0.5 & 0.5 & 0.5 & 0.5 & 0.5 & 0.5 \\
\hline & $\mathrm{MIC}_{90}$ & 1 & 2 & 1 & 1 & 2 & 1 & 2 & 2 & 2 & 2 \\
\hline & $\% \mathrm{~S}$ & 96.0 & 93.2 & 96.4 & 96.3 & 93.7 & 98.0 & 94.8 & 94.7 & 97.3 & 95.3 \\
\hline
\end{tabular}

$\% \mathrm{~S}, \%$ susceptible, amoxi-clav amoxicillin-clavulanate, pip-taz piperacillin-tazobactam, carbapenems = imipenem/meropenem.

ceftriaxone, levofloxacin and piperacillin-tazobactam. There was a marked increase in the $\mathrm{MIC}_{90}$ for several antimicrobials against Klebsiella spp. collected from the Middle Atlantic region. In addition, there was a higher frequency (11\%) of K. pneumonia isolates that produced ESBL ( $\mathrm{n}=584$ ) compared with the numbers of ESBLproducing $E$. coli isolates, and the highest numbers of ESBL-producing $K$. pneumonia isolates were recovered from the Middle Atlantic region (17.7\%, 268/1518). The ESBL-producing isolates were mostly susceptible to amikacin (77.1\%) and carbapenems (74.0\%) and were highly susceptible (92.1\%) to tigecycline (Table 4 ).

\section{Serratia marcescens}

S. marcescens isolates were highly susceptible to cefepime (97.4\%), carbapenems (96.7\%), tigecycline (95.9\%), and levofloxacin (94.4\%) (Table 3). Amikacin ( $\mathrm{MIC}_{50} 2 \mathrm{mg} /$ $\mathrm{L}, \mathrm{MIC}_{90} 8 \mathrm{mg} / \mathrm{L}, 99.1 \%$ susceptible) and piperacillintazobactam $\left(\mathrm{MIC}_{50} 1 \mathrm{mg} / \mathrm{L}, \mathrm{MIC}_{90} 8 \mathrm{mg} / \mathrm{L}, \quad 95.5 \%\right.$ susceptible) were also active, but susceptibilities to ceftriaxone and minocycline varied among the census regions. For example, there were considerably higher numbers of ceftriaxone-resistant isolates recovered from the East North Central and Middle Atlantic region when compared with recovery in New England $(78.3 \%$ and $77.8 \%$ versus $92.5 \%$ susceptible). In addition, resistance to minocycline was most prevalent in the West South Central region (73.5\% susceptible).

\section{Enterobacter spp.}

Enterobacter spp. were highly susceptible to amikacin (98.9\%), carbapenems (97.6\%), and cefepime (96.1\%), and tigecycline $\left(\mathrm{MIC}_{50} 0.5 \mathrm{mg} / \mathrm{L}, \mathrm{MIC}_{90} \leq 2 \mathrm{mg} / \mathrm{L}, 95.3 \%\right)$ was also highly effective (Table 3 ). In contrast, susceptibility to ceftriaxone, levofloxacin, and minocycline was more variable, and the differences were most pronounced when comparing $\mathrm{MIC}_{90}$ values. For example, isolates from the Pacific region were highly susceptible $(79.7 \%)$ to ceftriaxone, while organisms from the Mountain region were considerably more resistant (63.5\% susceptibility).

\section{Discussion}

Antimicrobial resistance among Gram-negative organisms continues to be a major concern, especially considering the potential for the rapid spread of resistance mechanisms and the limited treatment options that result. In this study, we examined the activity of $\beta$-lactam, aminoglycoside, and fluoroquinolone antimicrobials against Enterobacteriaceae 
Table 4 Antimicrobial susceptibility for ESBL-positive Escherichia coli and Klebsiella pneumoniae

\begin{tabular}{|c|c|c|c|}
\hline & & $\begin{array}{c}\text { ESBL-producing } \\
\text { E. coli }\end{array}$ & $\begin{array}{l}\text { ESBL-producing } \\
\text { K. pneumoniae }\end{array}$ \\
\hline & & $N=277$ & $N=584$ \\
\hline \multirow[t]{3}{*}{ Amikacin } & $\mathrm{MIC}_{50}$ & 4 & 16 \\
\hline & $\mathrm{MIC}_{90}$ & 16 & 32 \\
\hline & $\% \mathrm{~S}$ & 94.9 & 77.1 \\
\hline \multirow[t]{3}{*}{ Amoxi-clav } & $\mathrm{MIC}_{50}$ & 16 & 16 \\
\hline & $\mathrm{MIC}_{90}$ & $\geq 64$ & $\geq 64$ \\
\hline & $\% \mathrm{~S}$ & 29.6 & 29.1 \\
\hline \multirow[t]{3}{*}{ Ampicillin } & $\mathrm{MIC}_{50}$ & $\geq 64$ & $\geq 64$ \\
\hline & $\mathrm{MIC}_{90}$ & $\geq 64$ & $\geq 64$ \\
\hline & $\% \mathrm{~S}$ & 0.7 & 0.0 \\
\hline \multirow[t]{3}{*}{ Carbapenems } & $\mathrm{MIC}_{50}$ & $\leq 0.06$ & 0.12 \\
\hline & $\mathrm{MIC}_{90}$ & 0.25 & 16 \\
\hline & $\% \mathrm{~S}$ & 98.2 & 74.0 \\
\hline \multirow[t]{3}{*}{ Cefepime } & $\mathrm{MIC}_{50}$ & 32 & 16 \\
\hline & $\mathrm{MIC}_{90}$ & $\geq 64$ & $\geq 64$ \\
\hline & $\% \mathrm{~S}$ & 34.3 & 48.1 \\
\hline \multirow[t]{3}{*}{ Ceftriaxone } & $\mathrm{MIC}_{50}$ & $\geq 128$ & 64 \\
\hline & $\mathrm{MIC}_{90}$ & $\geq 128$ & $\geq 128$ \\
\hline & $\% \mathrm{~S}$ & 2.2 & 3.1 \\
\hline \multirow[t]{3}{*}{ Levofloxacin } & $\mathrm{MIC}_{50}$ & $\geq 16$ & $\geq 16$ \\
\hline & $\mathrm{MIC}_{90}$ & $\geq 16$ & $\geq 16$ \\
\hline & $\% \mathrm{~S}$ & 5.8 & 19.9 \\
\hline \multirow[t]{3}{*}{ Minocycline } & $\mathrm{MIC}_{50}$ & 4 & 4 \\
\hline & $\mathrm{MIC}_{90}$ & $\geq 32$ & $\geq 32$ \\
\hline & $\% \mathrm{~S}$ & 61.0 & 54.8 \\
\hline \multirow[t]{3}{*}{ Pip-taz } & $\mathrm{MIC}_{50}$ & 4 & 128 \\
\hline & $\mathrm{MIC}_{90}$ & 128 & $\geq 256$ \\
\hline & $\% \mathrm{~S}$ & 79.4 & 40.1 \\
\hline \multirow[t]{3}{*}{ Tigecycline } & $\mathrm{MIC}_{50}$ & 0.25 & 1 \\
\hline & $\mathrm{MIC}_{90}$ & 0.5 & 2 \\
\hline & $\% \mathrm{~S}$ & 100 & 92.1 \\
\hline
\end{tabular}

$\% \mathrm{~S}$ \% susceptible, amoxi-clav amoxicillin-clavulanate, pip-taz piperacillintazobactam, carbapenems $=$ imipenem $/$ meropenem.

and $A$. baumannii isolates collected from nine regions within the USA. We also examined the susceptibility of each isolate to tigecycline, a glycylcycline licensed to treat infections caused by a broad spectrum of microorganisms, many of which have acquired resistance to treatment with traditional antimicrobials. In addition, Halstead et al. [2007] published a comprehensive report of antimicrobial susceptibilities of Gram-negative isolates collected from the USA during 2004 and 2005 [8] and we extend the study by determining the antimicrobial susceptibilities of a more diverse group of isolates that highlights ongoing nationwide changes in resistance patterns. It should be noted, however, that we failed to test each appropriate Gram-negative isolate for susceptibility to imepenem and meropenem, which forced us to incorporate our findings into the broader category of carbapenem resistance. However, we are confident this shortcoming did not prevent valid comparison of our results with previous findings.

Several other recent studies also determined the susceptibilities of Gram-negative organisms to multiple antimicrobial agents $[2,12,13]$, with results similar to this study. For example, we detected similarly high prevalence of sensitivity of $K$. oxytoca and non-ESBL producing E. coli to levofloxacin, piperacillin-tazobactam, and ceftriaxone. In addition, Enterobacter spp., K. oxytoca, and $S$. marcescens were highly susceptible to the carbapenems, while the non-ESBL-producing E. coli and $K$. pneumoniae isolates were almost universally susceptible to the carbapenems. However, small numbers of carbapenem-resistant organisms were recovered from each genus, which also supports previous findings that highlight the necessity for continued monitoring for carbapenem resistance. In addition, A. baumannii isolates that were highly resistant to multiple other antimicrobial agents were also highly resistant to the carbapenems (imipenem/meropenem), a result which has been previously reported [14]. This is especially disconcerting since the only option for effective treatment of these highly resistant organisms, especially MDR A. baumannii infections, may be dependent on salvage agents such as colistin which introduce a host of additional complications $[14,15]$.

Comparing the susceptibility patterns to previous findings [8] also revealed several important trends. Most notably, the prevalence of resistant organisms remained essentially unchanged in the East South Central, Middle Atlantic, and Pacific regions; the prevalence of organisms that were resistant to levofloxacin also decreased significantly $(\mathrm{p}<0.01)$. Significant $(\mathrm{p}<0.01)$ increases in susceptibility were identified in 8 region/organism/antimicrobial agent combinations between 2004-2005 and 2005-2011, 4 of these occurring in the Middle Atlantic region. Significant decreases in susceptibility were noted in 26 cases over the same time interval; 12 of these occurred in East North Central while 8 were noted in South Atlantic. Notably, in South Atlantic, K. pneumoniae susceptibility to levofloxacin, amikacin, amoxicillin-clavulanate, cefepime, minocycline and piperacillin-tazobactam decreased significantly. In East North Central, A. baumannii susceptibility to amikacin, ceftriaxone, levofloxacin, minocycline and piperacillin-tazobactam reduced significantly while $E$. coli susceptibility to amoxicillin-clavulanate, cefepime, levofloxacin and minocycline decreased significantly. These findings highlight the importance of local efforts to monitor changing antimicrobial susceptibility patterns for 
accurately guiding appropriate treatment regimens. In addition, evaluating the infection control practices in regions where the prevalence of antibiotic resistant organisms has not increased significantly may provide important insight into effective methods for curbing emerging resistance in other regions.

Finally, despite the lack of established efficacy standards for predicting the success of treatment with tigecycline, our findings confirmed and extended previous observations of high in vitro activity against Enterobacteriaceae (E. coli, 100\% susceptible; Enterobacter, 98.4\% susceptible; ESBL-positive K. pneumoniae, 97.9\%) and also $A$. baumannii (94.4\% susceptible at $\leq 2 \mathrm{mg} / \mathrm{L}$ ) [16]. Therefore, additional studies to determine the efficacy of tigecycline in vivo, especially for treating infections with MDR organisms, are warranted.

\section{Competing interests}

GAD: is a paid investigator for the Tigecycline Evaluation and Surveillance Trial.

SMC: Gundersen Health System received modest monetary compensation from Pfizer Inc. for providing MIC values of isolates collected in-house. However, SMC received no direct financial support and also has no industrial/commercial relationships that may be interpreted to pose a conflict of interest.

MJD: is an employee of Pfizer Inc.

\section{Author's contributions}

GAD was involved in the collection of data for this study, the analysis and interpretation of these data, and in drafting and revising the content of this manuscript. GAD has given approval for this version of the manuscript to be published. SMC was involved in the collection of data for this study, the analysis and interpretation of these data, and in drafting and revising the content of this manuscript. SMC has given approval for this version of the manuscript to be published. MD was involved in the concept, design and execution of the T.E.S.T. study. MD has also been involved in the revision of this manuscript, and given approval for this version of the manuscript to be published. All authors read and approved the final manuscript.

\section{Acknowledgements}

T.E.S.T. is funded by Pfizer Inc.

The authors thank the investigators and laboratories from each region for their participation and the IHMA staff for coordinating the study. No authors were paid for their contributions to this manuscript.

Special thanks also to Dr. Wendy Hartley and Dr. Rod Taylor (Micron Research Ltd, Ely, UK) for expert assistance with medical writing and Micron Research Ltd for data management services. Dr. Wendy Hartley and Dr. Rod Taylor (Micron Research Ltd, Ely, UK) provided medical writing services, which were funded by Pfizer Inc. Micron Research Ltd also provided data management services which were funded by Pfizer Inc.

\section{Author details}

'Indiana University Health Pathology Laboratory, Indianapolis 46202, IN, USA. ${ }^{2}$ Microbiology Research and Molecular Diagnostics Laboratory, Gundersen Health System, La Crosse, Wisconsin 54601, USA. ${ }^{3}$ Pfizer Inc, 500 Arcola Road, E-Dock, Collegeville 19426, PA, USA.

Received: 14 May 2013 Accepted: 1 September 2013

Published: 5 September 2013

\section{References}

1. Mera RM, Miller LA, Amrine-Madsen H, Sahm DF: Acinetobacter baumannii 2002-2008: increase of carbapenem-associated multiclass resistance in the United States. Microb Drug Resist 2010, 16:209-215.

2. Davies TA, Queenan AM, Morrow BJ, Shang W, Amsler K, He W, Lynch AS, Pillar C, Flamm RK: Longitudinal survey of carbapenem resistance and resistance mechanisms in enterobacteriaceae and non-fermenters from the USA in 2007-09. J Antimicrob Chemother 2011, 66:2298-2307.

3. Mauldin PD, Salgado CD, Hansen IS, Durup DT, Bosso JA: Attributable hospital cost and length of stay associated with health care-associated infections caused by antibiotic-resistant gram-negative bacteria. Antimicrob Agents Chemother 2010, 54:109-115.

4. Zarkotou O, Pournaras S, Tselioti P, Dragoumanos V, Pitiriga V, Ranellou K, Prekates A, Themeli-Digalaki K, Tsakris A: Predictors of mortality in patients with bloodstream infections caused by KPC-producing Klebsiella pneumoniae and impact of appropriate antimicrobial treatment. Clin Microbiol Infect 2011, 17:1798-1803.

5. Shorr AF, Micek ST, Welch EC, Doherty JA, Reichley RM, Kollef MH: Inappropriate antimicrobial therapy in gram-negative sepsis increases hospital length of stay. Crit Care Med 2011, 39:46-51.

6. Pankey GA: Tigecycline. J Antimicrob Chemother 2005, 56:470-480

7. Pfizer Inc (Wyeth Pharmaceuticals): Tygaci ${ }^{\oplus}$ Product Insert. Philadelphia, PA, USA. Last Revised January 2011 [http://www.pfizerpro.com/hcp/tygacil]

8. Halstead DC, Abid J, Dowzicky MJ: Antimicrobial susceptibility among Acinetobacter calcoaceticus-baumannii complex and Enterobacteriaceae collected as part of the Tigecycline Evaluation and Surveillance Trial. J Infect 2007, 55:49-57.

9. Clinical and Laboratory Standards Institute (CLSI): Methods for dilution antimicrobial susceptibility tests for bacteria that grow aerobically; Approved standard - 8th edition. Document M7-A8. Wayne, PA: CLSI; 2009. ISBN 1-56238-689-1.

10. Clinical and Laboratory Standards Institute (CLSI): Performance standard for antimicrobial susceptibility testing; 20th Ed. Document M100-S20. Wayne, PA: CLSI; 2010.

11. Clinical and Laboratory Standards Institute (CLSI): Performance standard for antimicrobial susceptibility testing, 20th Ed (June 2010 update). Document M100S20U. Wayne, PA: CLSI; 2010.

12. Flamm RK, Sader HS, Farrell DJ, Jones RN: Summary of ceftaroline activity against pathogens in the United States, 2010: report from the Assessing Worldwide Antimicrobial Resistance Evaluation (AWARE) surveillance program. Antimicrob Agents Chemother 2012, 56:2933-2940.

13. Gupta N, Limbago BM, Patel JB, Kallen AJ: Carbapenem-resistant Enterobacteriaceae: epidemiology and prevention. Clin Infect Dis 2011 53:60-67.

14. Adams-Haduch JM, Onuoha EO, Bogdanovich T, Tian GB, Marschall J, Urban CM, Spellberg BJ, Rhee D, Halstead DC, Pasculle AW, Doi Y: Molecular epidemiology of carbapenem-nonsusceptible Acinetobacter baumannii in the United States. J Clin Microbiol 2011, 49:3849-3854.

15. Esterly JS, Griffith M, Qi C, Malczynski M, Postelnick MJ, Scheetz MH: Impact of carbapenem resistance and receipt of active antimicrobial therapy on clinical outcomes of Acinetobacter baumannii bloodstream infections. Antimicrob Agents Chemother 2011, 55:4844-4849.

16. Sader HS, Farrell DJ, Jones RN: Tigecycline activity tested against multidrugresistant Enterobacteriaceae and Acinetobacter spp. isolated in US medical centers (2005-2009). Diagn Microbiol Infect Dis 2011, 69:223-227.

doi:10.1186/1476-0711-12-24

Cite this article as: Denys et al:: Antimicrobial susceptibility among gram-negative isolates collected in the USA between 2005 and 2011 as part of the Tigecycline Evaluation and Surveillance Trial (T.E.S.T.). Annals of Clinical Microbiology and Antimicrobials 2013 12:24.

\section{Submit your next manuscript to BioMed Central and take full advantage of:}

- Convenient online submission

- Thorough peer review

- No space constraints or color figure charges

- Immediate publication on acceptance

- Inclusion in PubMed, CAS, Scopus and Google Scholar

- Research which is freely available for redistribution 